\title{
Quantitative Bone Marrow MRI in Children with Acute Lymphoblastic Leukemia
}

\author{
Galina V. Tereshchenko ${ }^{1}$; Nataliia A. Kriventsova ${ }^{1 *}$; Dmitry A. Kupriyanov ${ }^{1,2}$; \\ Peter E. Menshchikov ${ }^{1,2}$; Dmitry V. Litvinov ${ }^{1}$; Galina A. Novichkova ${ }^{1}$ \\ ${ }^{1}$ Dmitry Rogachev National Medical Research Center of \\ Pediatric Hematology, Oncology and Immunology, Moscow, Russia \\ ${ }^{2}$ Philips Healthcare, Moscow, Russia
}

\begin{abstract}
The aim of this study was to evaluate, using MRI, the changes in bone marrow fat fraction (BMFF) of patients with acute lymphoblastic leukemia (ALL), in comparison with children without hematological disorders.

Methods and Results: The cohort of the study subjects included 20 patients aged between 5 and 17 years (mean age of $11.2 \pm 3.6$ years; 10 boys and 10 girls) with clinically and morphologically confirmed diagnosis of ALL All patients underwent MRI scanning in the acute phase of the disease before the start of specific therapy. Then, the study was repeated in 10 patients (mean age of $12.2 \pm 2.3$ years; 8 boys and 2 girls) during treatment, according to the ALL-MB 2015 protocol for patients with primary ALL and according to the ALL-REZ-MB 2016 protocol for patients with relapsed ALL. The control group consisted of 24 healthy controls of the same age group (mean age of $12 \pm 2.8$ years; 17 boys and 7 girls) with no prior hematologic diseases. MRI scanning was carried out using a Philips Achieva dStream 3T scanner with a 32-channel FlexCoverage abdominal receiving coil. The MRI protocol included images obtained with the mDIXON Quant technique in the coronal plane, completely covering the pelvic bones and lumbar spine. Fat fraction maps were generated automatically on the MRI console using the 7-peak fat model and were corrected for T2* effects. Regions of interest (ROI) measuring $150 \mathrm{~mm}^{2}$ were placed in the bodies of the left and right iliac bones (Ilium L, Ilium R) as well as in the L4 and L5 vertebral bodies, taking care to avoid blood vessels, cortical bones and areas that could potentially contain artifacts.

In the group of healthy controls, BMFF value was $51 \pm 11 \%$ in the bodies of the iliac bones and $32 \pm 10 \%$ in the lumbar vertebrae. In the group of patients with the acute phase of the disease, BMFF was as low as $3.1 \pm 2.6 \%$ in all the bone structures. In patients who had undergone chemotherapy, the mean BMFF increased up to $77 \pm 7 \%$ in the iliac bones and up to $65 \pm 13 \%$ in the vertebrae. Student's t-test for dependent samples revealed a statistically significant increase in the mean BMFF values in all the bone structures after chemotherapy $(P<0.01)$. After schemotherapy, BMFF was also significantly higher than under normal conditions $(P<0.01)$.

Conclusion: This study provides important diagnostic information for various phases of ALL treatment, especially in suspected cases of resistant or recurrent disease. (International Journal of Biomedicine. 2021;11(2):141-145.)
\end{abstract}

Key Words: bone marrow $\bullet$ quantitative magnetic resonance imaging $\bullet$ pediatrics $\bullet$ hematology $\bullet$ fat fraction $\bullet$ fat cells

For citation: Tereshchenko GV, Kriventsova NA, Kupriyanov DA, Menshchikov PE, Litvinov DV, Novichkova GA. Quantitative Bone Marrow MRI in Children with Acute Lymphoblastic Leukemia. International Journal of Biomedicine. 2021;11(2):141-145. doi:10.21103/Article11(2)_OA3

\section{Abbreviations}

ALL, acute lymphoblastic leukemia; BM, bone marrow; BMFF, bone marrow fat fraction; FF, fat fraction.

\section{Introduction}

Acute lymphoblastic leukemia (ALL) is a cancer of the red bone marrow (BM). ${ }^{(1)}$ As of 2017, the incidence rate of leukemia was 4.5 per 100,000 children (4.98 among boys, 4.18 among girls) aged between 0 and 14 years. According to an analysis of the data from 2007 to 2017, the incidence rate of leukemia in this age group increased by $20.28 \%$ (the average annual growth rate $-1.82 \%)$. $^{(2)}$ Modern treatment protocols allow us to achieve a cure in up to $95 \%$ of young patients; however the treatment is long and expensive. Due to the slow but still steady growth in the number of new cases year by 
year, there is a clear need for a multimodal approach to the primary prevention and diagnosis of malignant hematological disorders in children. ${ }^{(3)}$

Historically, two types of BM have been distinguished: "red" (hematopoietic) and "yellow" (fatty). Cancer researchers delineate an important role of BM adipocytes in oncohematology. Adipocytes contribute to the development and the continued growth of tumors, the metastasis to the BM and the development of resistance to chemotherapy. ${ }^{(4,5)}$ Adipocytes can be studied using not only laboratory methods, but also MRI, which allows us to assess the total number of adipocytes in the BM..$^{(6,7)}$ It has been shown that all the sequences have high sensitivity to neoplastic and inflammatory changes in the BM of children. ${ }^{\left({ }^{8}\right)}$

At different stages of ALL treatment approaches, the assessment of "red"(hematopoietic)-to-"yellow"(fat) ratio in the BM, using MRI, can provide additional information about the condition of the BM during the conducted polychemotherapy. ${ }^{(9)}$

The aim of this study was to evaluate, using MRI, the changes in BMFF of patients with ALL, in comparison with children without hematological disorders.

\section{Materials and Methods}

This study was approved by the Independent Ethics Committee of the D. Rogachev NMRCPHOI, supported by the Scientific Council of the D. Rogachev NMRCPHOI of the Ministry of Healthcare of the Russian Federation and complies with the World Medical Association Code of Ethics for medical research involving human subjects. Written informed consent was obtained from patients and their parents.

The cohort of the study subjects included 20 patients aged between 5 and 17 years (mean age of $11.2 \pm 3.6$ years; 10 boys and 10 girls) with clinically and morphologically confirmed diagnosis of ALL, who underwent treatment at the D. Rogachev NMRCPHOI from October 2017 to March 2020. All patients underwent MRI scanning in the acute phase of the disease before the start of specific therapy (Table 1). Then, the study was repeated in 10 patients (mean age of $12.2 \pm 2.3$ years, 8 boys and 2 girls) during treatment, according to the ALL-MB 2015 protocol for patients with primary ALL and according to the ALL-REZ-MB 2016 protocol for patients with relapsed ALL (Table 2).

\section{Table 1.}

The number of patients in the acute phase of ALL before the start of chemotherapy. The first acute period is the acute phase of the disease in a patient with newly diagnosed $A L L$; the second acute period is the acute phase of the disease in a patient with relapsed $A L L$.

\begin{tabular}{|l|c|c|c|c|}
\hline & \multicolumn{4}{|c|}{ White blood cells $\left(\times 10^{9} / \mathrm{L}\right)$} \\
\hline & $\begin{array}{c}\text { Number } \\
\text { of patients }\end{array}$ & $<6.05$ & $6.05-9.8$ & $>9.8$ \\
\hline First acute period & 11 & 4 & 2 & 5 \\
\hline Second acute period & 9 & 2 & 1 & 6 \\
\hline
\end{tabular}

The control group consisted of 24 healthy controls of the same age group (mean age of $12 \pm 2.8 ; 17$ boys and 7 girls) with no prior hematologic diseases.
Table 2.

The number of patients with an indication of the treatment stage and the protocol according to which they received treatment.

\begin{tabular}{|c|c|c|}
\hline Treatment protocol & $\begin{array}{c}\text { Number of } \\
\text { patients }\end{array}$ & Treatment stage \\
\hline ALL-MB 2015 & 5 & After cycle F1, F2 \\
\hline ALL REZ MB 2016 & 5 & After cycle F1,2 R1,2 \\
\hline
\end{tabular}

\section{$\underline{\text { MRI protocol }}$}

MRI scanning was carried out using a Philips Achieva dStream 3T scanner (Philips Healthcare, Best, the Netherlands) with a 32-channel FlexCoverage abdominal receiving coil. The MRI protocol included images obtained with the mDIXON Quant technique in the coronal plane, completely covering the pelvic bones and lumbar spine. mDIXON images were registered with the following parameters of a gradient multiecho sequence: repetition time $(\mathrm{TR})=11 \mathrm{~ms}, 6$ times to echo (TE) - minimal TE (TE1) $=1.2 \mathrm{~ms}$, with an interval $(\Delta \mathrm{TE})$ $=0.8 \mathrm{~ms}$; flip angle $(\mathrm{FA})-3^{\circ}$ to minimize the influence of $\mathrm{T} 1$ weighting; the number of slices -35 , slice thickness $-4 \mathrm{~mm}$, with a gap $--2 \mathrm{~mm}$; field of view $(\mathrm{FOV})=360 \times 360 \mathrm{~mm}^{2}$, voxel size $-3.5 \times 3.5 \times 4.0 \mathrm{~mm}$ for registration and $2.5 \times 2.5 \times 2.0 \mathrm{~mm}$ for reconstruction; the frequency-encoding direction - $\mathrm{A} / \mathrm{P}$ (to minimize respiratory motion artifacts); the SENSE acceleration factor -2 . The scan time was $1 \mathrm{~min}$.

The fat fraction (FF) was calculated as the ratio between fat signal intensity $\left(\mathrm{S}_{\mathrm{fat}}\right)$ and water signal intensity $\left(\mathrm{S}_{\mathrm{H} 20}\right)$ using the following formula:

Fat fraction $(F F)=\frac{S(\text { fat })}{S(\text { fat })+S(H 2 O)}$

FF maps were generated automatically on the MRI console using the 7-peak fat model and were corrected for T2* effects. ${ }^{(10)}$

Regions of interest (ROI) measuring $150 \mathrm{~mm}^{2}$ were placed in the bodies of the left and right iliac bones (Ilium L, Ilium R) as well as in the L4 and L5 vertebral bodies, taking care to avoid blood vessels, cortical bones and areas that could potentially contain artifacts (Fig.1).

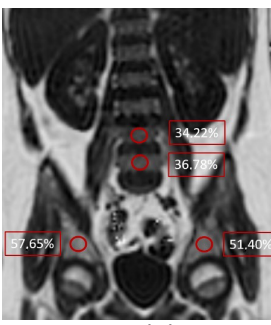

(a)

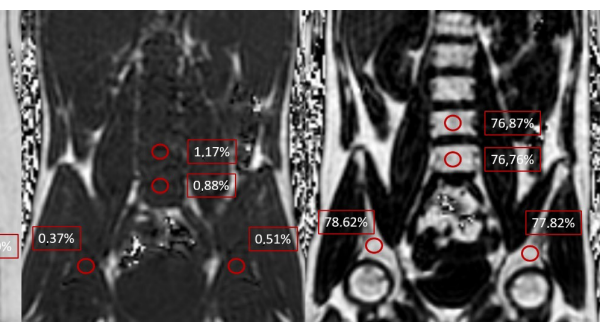

(b) (c)

Fig. 1. FF maps with regions of interest and mean values measured in these regions. (a) A healthy control. (b) A patient diagnosed with ALA, before the start of chemotherapy. (c) The same patient after the first chemotherapy cycle.

Statistical analysis was performed using Jamovi 1.1.9.0.25. The Shapiro-Wilk W test was used in testing for normality. For data with normal distribution, inter-group comparisons were performed using Student's t-test. The Mann-Whitney U Test was used to compare the differences between the two independent groups (for nonparametric data). 
The Wilcoxon criterion was used to compare the differences between the paired samples. A probability value of $P<0.05$ was considered statistically significant.

\section{Results}

The Mann-Whitney U test did not show any significant differences between the groups of patients with respect to age $(P=0.854)$. The BMFF values during the first and the second acute periods were compared within the "before specific therapy" group of patients $(n=20)$ Student's t-test did not reveal any significant differences between the values $(P=0.962)$. In the "after chemotherapy" group $(\mathrm{n}=10)$, the data were compared in patients who had been treated in accordance with the ALL-MB 2015 protocol and those who had received treatment under the ALL-REZ-MB 2016 protocol. The MannWhitney $U$ test did not show any significant differences between the values $(P=0.753)$.

In the group of healthy controls, BMFF value was $51 \pm 11 \%$ in the bodies of the iliac bones and $32 \pm 10 \%$ in the lumbar vertebrae. All patients in the acute phase of the disease were included in one group. In these patients, BMFF was as low as $3.1 \pm 2.6 \%$ in all the bone structures. All patients who had undergone treatment were included in another group. In this group, the mean BMFF increased up to $77 \pm 7 \%$ in the iliac bones and up to $65 \pm 13 \%$ in the vertebrae. The BMFF values measured at several sites in the three groups of patients are summarized in Table 3.

Table 3.

Fat fraction in the three groups of study patients in the ROI

\begin{tabular}{|c|c|c|c|c|c|}
\hline \multirow{2}{*}{\multicolumn{2}{|c|}{ Group }} & \multicolumn{4}{|c|}{ Fat fraction $($ mean $\pm \mathrm{SD}, \%)$} \\
\hline & & Ilium L & Ilium R & L4 & L5 \\
\hline \multicolumn{2}{|c|}{$\begin{array}{l}\text { Healthy controls } \\
\qquad(\mathrm{n}=24)\end{array}$} & $52 \pm 11$ & $50 \pm 12$ & $31 \pm 9$ & $32 \pm 12$ \\
\hline \multirow{4}{*}{$\begin{array}{l}\text { Patients with } \\
\text { ALL }\end{array}$} & $\begin{array}{l}\text { The acute } \\
\text { phase of the } \\
\text { disease } \\
(n=20)\end{array}$ & $3.2 \pm 2.7$ & $3.7 \pm 3.3$ & $2.6 \pm 1.9 *$ & $2.9 \pm 2.5$ \\
\hline & $P$-value & $P<0.01 *$ & $P<0.01^{*}$ & $P<0.01 *$ & $P<0.01 *$ \\
\hline & $\begin{array}{l}\text { After } \\
\text { chemotherapy } \\
(n=10)\end{array}$ & $78 \pm 9$ & $78 \pm 7$ & $64 \pm 13$ & $66 \pm 13$ \\
\hline & $P$-value & $P<0.01^{\wedge}$ & $P<0.01^{\wedge}$ & $P<0.01^{\wedge}$ & $P<0.01^{\wedge}$ \\
\hline
\end{tabular}

*- between healthy controls and acute phase of ALL

$\wedge$ - between healthy controls and after chemotherapy; between acute phase of ALL and after chemotherapy

Under normal conditions, the mean fat content in the iliac bones was statistically significantly higher than in the L4 and L5 vertebrae $(P<0.01)$. In the acute phase of the disease, $\mathrm{BMFF}$ values were statistically significantly lower than under normal conditions $(P<0.01)$. Student's t-test for dependent samples revealed a statistically significant increase in the mean BMFF values in all the bone structures after chemotherapy $(P<0.01)$. After specific therapy, BMFF was also significantly higher than under normal conditions $(P<0.01)$ (Fig.2).

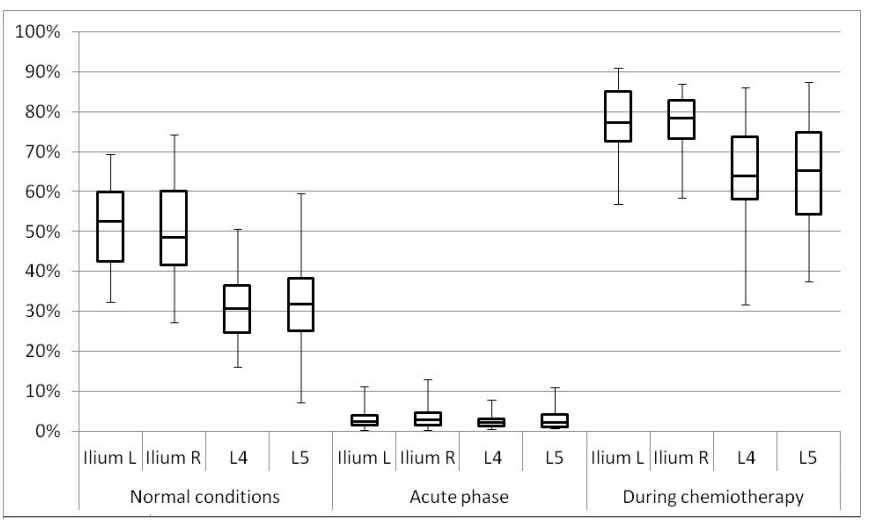

Fig. 2. A chart showing the range of fat fraction values (\%) in the three groups of patients in the four regions of interest.

\section{Discussion}

Malignant leukemic processes in the BM affect the cellularity and composition of the BM, which inevitably leads to changes in signal intensity of bone structures on MRI. ${ }^{(11)}$ In healthy adolescents, bones have a moderately hyperintense signal on T1-weighted (T1WI) and T2-weighted (T2WI) images, as compared to the surrounding muscles. In case of malignant transformation of the BM, signal intensity on T1WI and T2WI is similar to that of the surrounding muscles. After chemotherapy, the signal intensity of the BM on T1WI and T2WI becomes significantly higher than the signal intensity of the muscles, and is then comparable to that of the subcutaneous fat (Fig.3). Although visual assessment is more applicable in a clinical setting, it is not objective and cannot provide us with any quantitative data, so it may not be sufficient to evaluate $\mathrm{BM}$ involvement at the time of investigation.

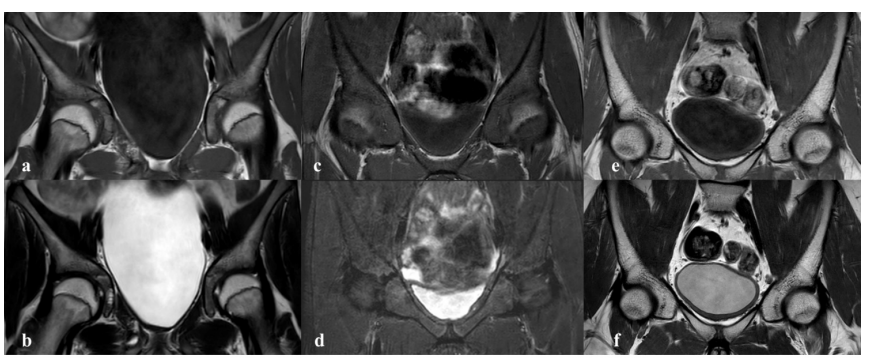

Fig. 3. Pelvic bone MRI in the coronal plane. (a-b) A healthy control without any hematologic diseases, T1WI and T2WI; (cd) A female patient in the acute phase of ALA before treatment, TIWI and T2WI/STIR; (e-f) A male patient undergoing chemotherapy, T1WI and T2WI.

The signal intensity (SI) of BM is different on T1W images for the patients with hematological disorders and for the healthy control group. ${ }^{(12)}$ Assessment of the BMFF, defined as a ratio of the fat content to the water content within a chosen area, can be used as a biomarker of changes in the BM.(7)

In this study, we used a multiple echo DIXON FFE sequence (mDIXON Quant) for the quantification of fat and water. There are several factors influencing the accuracy of tissue fat quantification, such as fast $\mathrm{T}_{2} *$ signal decay, field inhomogeneities, the complexity of the spectral model of fat, 
and T1 effects. The mDIXON-Quant sequence with multiple echoes helps us to avoid these confounding factors by using a low flip angle to minimize influence of T1 effects and by taking into account spectral complexity of the fat signal model and $\mathrm{T} 2 *$ correction. $^{(13,14)}$

According to the international guidelines, the preferred site for BM aspiration in children is the iliac crest. For this reason, we used a large field of view (FOV), which covered both iliac bones. ${ }^{(15)}$ Since in a number of articles on BM examination vertebrae were chosen as a region of interest, we also included the lumbar spine in the FOV. ${ }^{(16)}$

BM that changes drastically during a person's life is very susceptible to pathological processes occurring in the body. Starting from prenatal development and up to the age of 30 , red BM is gradually being replaced by yellow BM. ${ }^{(16)}$ In order to avoid unreliable results, the healthy controls and the patients included in the study were of the same age.

In the group of controls, the fat content in the vertebrae was, on average, $20 \%$ lower than in the iliac bones. This may be associated with a higher BM cellularity in the vertebrae and fatty bone marrow conversion in children and adolescents, which occurs in the vertebrae and ribs much later than in other bones. ${ }^{(17)}$

MRI for the measurement of BMFF is applied in a number of hematologic and bone disorders: to assess focal changes in bones, ${ }^{(18)}$ in osteoporosis, ${ }^{(19)}$ in Gaucher disease, ${ }^{(20)}$ and to evaluate treatment response in patients with myelofibrosis. ${ }^{(21)}$ The majority of studies include only adult patients; therefore, there is a lack of data on children. In the literature, there is only one pilot study, which included four patients with ALL before the initiation of a specific therapy. ${ }^{(22)}$ Samet et al. ${ }^{(22)}$ showed that BMFF in children with ALL was below $10 \%$, which correlates with our results. Our study included more subjects and the BM assessment was performed not just before the initiation of treatment but also during chemotherapy.

This study demonstrated that the BM water-fat ratios in children with ALL vary depending on clinical status and significantly differ from normal values. A decrease in BMFF below $10 \%$ in patients in the acute phase of the disease may be associated with the total infiltration of the BM by abnormal cells, and the displacement of adipocytes. ${ }^{(23)}$ Cytoreductive chemotherapy significantly reduces the number of both abnormal and normal cells in the BM, resulting in "exhaustion" of BM. Hematopoietic cells are replaced by adipocytes, and at that time, the BMFF increases to over $70 \%{ }^{(5)}$

In conclusion, quantitative MRI assessment of BMFF is a promising technique, allowing for the assessment of BM cellularity as a longitudinal study. This study provides important diagnostic information for various phases of ALL treatment, especially in suspected cases of resistant or recurrent disease.

\section{Competing Interests}

The authors declare that they have no competing interests.

*Corresponding author: Nataliia A. Kriventsova. Dmitry Rogachev National Medical Research Center of Pediatric Hematology, Oncology and Immunology. Moscow, Russia. E-mail: nataliya. krivencova@fccho-moscow.ru

\section{References}

1. Davis AS, Viera AJ, Mead MD. Leukemia: an overview for primary care. Am Fam Physician. 2014 May 1;89(9):731-8.

2. Kaprin AD, Starinsky VV, Petrova G V. Malignant neoplasms in Russia in 2017. Moscow; 2018. Available from: https://glavonco.ru/upload/pages/cancer-register/statistika zabol_2017.pdf

3. Whitehead TP, Metayer C, Wiemels JL, Singer AW, Miller MD. Childhood Leukemia and Primary Prevention. Curr Probl Pediatr Adolesc Health Care. 2016 Oct;46(10):317-352. doi: 10.1016/j.cppeds.2016.08.004.

4. Veldhuis-Vlug AG, Rosen CJ. Clinical implications of bone marrow adiposity. J Intern Med. 2018 Feb;283(2):121-139. doi: 10.1111/joim.12718.

5. Wang $\mathrm{H}$, Leng $\mathrm{Y}$, Gong $\mathrm{Y}$. Bone Marrow Fat and Hematopoiesis. Front Endocrinol (Lausanne). 2018 Nov 28;9:694. doi: 10.3389/fendo.2018.00694.

6. Karampinos DC, Ruschke S, Dieckmeyer M, Diefenbach M, Franz D, Gersing AS, Krug R, Baum T. Quantitative MRI and spectroscopy of bone marrow. J Magn Reson Imaging. 2018 Feb;47(2):332-353. doi: 10.1002/jmri.25769

7. Kriventsova NA, Kupriyanov DA, Menchshikov PE, and Terechshenko GV. MR-biomarker of bone marrow in children with acute lymphoblast leukemia. RJER. 2020;10(4):159-168. doi:10.21569/2222-7415-2020-10-4-159-168

8. Chan BY, Gill KG, Rebsamen SL, Nguyen JC. MR Imaging of Pediatric Bone Marrow. Radiographics. 2016 Oct;36(6):1911-1930. doi: 10.1148/rg.2016160056.

9. Foster K, Chapman S, Johnson K. MRI of the marrow in the paediatric skeleton. Clin Radiol. 2004 Aug;59(8):651-73. doi: 10.1016/j.crad.2004.02.001.

10. Lee SH, Yoo HJ, Yu SM, Hong SH, Choi JY, Chae HD. Fat Quantification in the Vertebral Body: Comparison of Modified Dixon Technique with Single-Voxel Magnetic Resonance Spectroscopy. Korean J Radiol. 2019 Jan;20(1):126-133. doi: 10.3348/kjr.2018.0174.

11. Chan BY, Gill KG, Rebsamen SL, Nguyen JC. MR Imaging of Pediatric Bone Marrow. Radiographics. 2016 Oct;36(6):1911-1930. doi: 10.1148/rg.2016160056.

12. Koyama H, Yoshihara H, Kotera M, Tamura T, Sugimura $\mathrm{K}$. The quantitative diagnostic capability of routine MR imaging and diffusion-weighted imaging in osteoporosis patients. Clin Imaging. 2013 Sep-Oct;37(5):925-9. doi: 10.1016/j.clinimag.2013.05.001.

13. Ma J. Dixon techniques for water and fat imaging. J Magn Reson Imaging. 2008 Sep;28(3):543-58. doi: 10.1002/ jmri.21492.

14. Karampinos DC, Melkus G, Baum T, Bauer JS, Rummeny EJ, Krug R. Bone marrow fat quantification in the presence of trabecular bone: initial comparison between water-fat imaging and single-voxel MRS. Magn Reson Med. 2014 Mar;71(3):1158-65. doi: 10.1002/mrm.24775.

15. Abla O, Friedman J, Doyle J. Performing bone marrow aspiration and biopsy in children: Recommended guidelines. Paediatr Child Health. 2008 Jul;13(6):499-501. doi: 10.1093/ pch/13.6.499.

16. Baum T, Rohrmeier A, Syväri J, Diefenbach MN, Franz $\mathrm{D}$, Dieckmeyer $\mathrm{M}$, et al. Anatomical Variation of AgeRelated Changes in Vertebral Bone Marrow Composition Using Chemical Shift Encoding-Based Water-Fat Magnetic Resonance Imaging. Front Endocrinol (Lausanne). $2018 \mathrm{Apr}$ 4;9:141. doi: 10.3389/fendo.2018.00141. 
17. Custer RP and Ahlfeldt FE. Studies on the structure and function of bone marrow: II. Variations in cellularity in various bones with advancing years of life and their relative response to stimuli. J Lab Clin Med. 1932;17(10):960-962.

18. Yoo HJ, Hong SH, Kim DH, Choi JY, Chae HD, Jeong BM, Ahn JM, Kang HS. Measurement of fat content in vertebral marrow using a modified dixon sequence to differentiate benign from malignant processes. J Magn Reson Imaging. 2017 May;45(5):1534-1544. doi: 10.1002/jmri.25496.

19. Chang R, Ma X, Jiang Y, Huang D, Chen X, Zhang M, Hao D. Percentage fat fraction in magnetic resonance imaging: upgrading the osteoporosis-detecting parameter. BMC Med Imaging. 2020 Mar 17;20(1):30. doi: 10.1186/s12880-020-00423-0.

20. Maas M, van Kuijk C, Stoker J, Hollak CE, Akkerman EM, Aerts JF, den Heeten GJ. Quantification of bone involvement in Gaucher disease: MR imaging bone marrow burden score as an alternative to Dixon quantitative chemical shift MR imaging--initial experience. Radiology. 2003 Nov;229(2):55461. doi: 10.1148/radiol.2292020296.

21. Luker GD, Nguyen HM, Hoff BA, Galbán CJ, Hernando D, Chenevert TL, et al. A Pilot Study of Quantitative MRI Parametric Response Mapping of Bone Marrow Fat for Treatment Assessment in Myelofibrosis. Tomography. 2016 Mar;2(1):67-78. doi: 10.18383/j.tom.2016.00115.

22. Samet JD, Deng J, Schafernak K, Arva NC, Lin X, Peevey J, Fayad LM. Quantitative magnetic resonance imaging for determining bone marrow fat fraction at $1.5 \mathrm{~T}$ and $3.0 \mathrm{~T}$ : a technique to noninvasively assess cellularity and potential malignancy of the bone marrow. Pediatr Radiol. 2021 Jan;51(1):94-102. doi: 10.1007/s00247-020-04809-8.

23. Samimi A, Ghanavat M, Shahrabi S, Azizidoost S, Saki N. Role of bone marrow adipocytes in leukemia and chemotherapy challenges. Cell Mol Life Sci. 2019 Jul;76(13):2489-2497. doi: 10.1007/s00018-019-03031-6. 